SELECT-MONOTHERAPY enrolled methotrexate (MTX)-IR pts receiving UPA $15 \mathrm{mg}$ or $30 \mathrm{mg}$ monotherapy or blinded MTX for $14 \mathrm{wks}$. After $12 / 14 \mathrm{wks}$, pts could enter a blinded long-term extension and receive UPA $15 \mathrm{mg}$ or $30 \mathrm{mg}$ for up to 5 years. This post hoc analysis evaluated clinical REM (CDAI $\leq 2.8$; SDAI $\leq 3.3$ ), LDA (CDAI $\leq 10$; SDAI $\leq 11)$, and DAS28(CRP) $<2.6 / \leq 3.2$ at first occurrence before Wk 84 ; additionally, these measures were evaluated at 3,6, and 12 months after the first occurrence for the total number of pts randomized to UPA $15 \mathrm{mg}$. Sustainability of response was evaluated by Kaplan-Meier only for those pts who achieved REM/ LDA and was defined as time to the earliest date of losing response at two consecutive visits or discontinuation of study drug. The predictive ability of time to clinical REM/LDA was assessed using Harrell's concordance (c)-index (for reference, an index $\sim 0.5$, indicates no ability to predict; an index of 1 or -1 would be a perfect prediction). The last follow up dates were 22 March, 2018 (SELECT-NEXT) and 25 May, 2019 (SELECT-MONOTHERAPY), when all pts had reached the Wk 84 visit. Results: Through Wk 84, the percent of treated pts achieving CDAI REM/LDA was $43 \% / 79 \%$ for those receiving UPA $15 \mathrm{mg}$ with background csDMARD(s) (SELECT-NEXT) and $37 \% / 76 \%$ for those receiving UPA $15 \mathrm{mg}$ without background csDMARD(s) (SELECT-MONOTHERAPY). 35\%/25\% of pts randomized to UPA $15 \mathrm{mg}$ with background $\operatorname{csDMARD}(\mathrm{s})$ and $27 \% / 23 \%$ of pts randomized to UPA $15 \mathrm{mg}$ without background csDMARD(s) achieved sustained CDAI REM through $6 / 12$ months after the first occurrence. $64 \% / 56 \%$ of pts randomized to UPA $15 \mathrm{mg}$ with background csDMARD(s) and $61 \% / 56 \%$ of pts randomized to UPA $15 \mathrm{mg}$ without background $\operatorname{csDMARD}(\mathrm{s})$ achieved sustained CDAI LDA through 6/12 months after the first occurrence (Figure 1). Time to initial clinical REM/LDA did not appear to be associated with sustained disease control. The c-indices $(95 \% \mathrm{Cl})$ for CDAI REM in the UPA $15 \mathrm{mg}$ with background csDMARD(s) and UPA $15 \mathrm{mg}$ without background csDMARD(s) groups were $0.541(0.47,0.62)$ and $0.568(0.49,0.65)$ and that of LDA were $0.521(0.46,0.58)$ and $0.498(0.43$ $0.56)$, respectively. Through last follow-up visit, $55 \%$ of pts receiving UPA $15 \mathrm{mg}$ with background csDMARD(s) and $62 \%$ of pts receiving UPA $15 \mathrm{mg}$ without background csDMARD(s) remained in CDAI REM while $72 \%$ and $70 \%$ of pts remained in CDAI LDA, respectively (Figure 2). Similar results were observed across other disease activity measures (SDAI REM/LDA and DAS28(CRP) $<2.6 / \leq 3.2$ ).

Conclusion: More than a quarter and more than a half of pts with RA and prior IR to csDMARD(s) receiving UPA with or without background csDMARD therapy achieved sustained clinical REM and LDA, respectively, across disease activity measures. Sustainability of responses appeared comparable among pts receiving UPA with or without background csDMARDs through up to 84 wks. References:

[1] EULAR: Smolen JS, et al. Ann Rheum Dis 2017;76:960-977.

[2]ACR: Singh et al. Arthritis \& Rheumatology Vol. 68, No. 1, January 2016, pp 1-26.
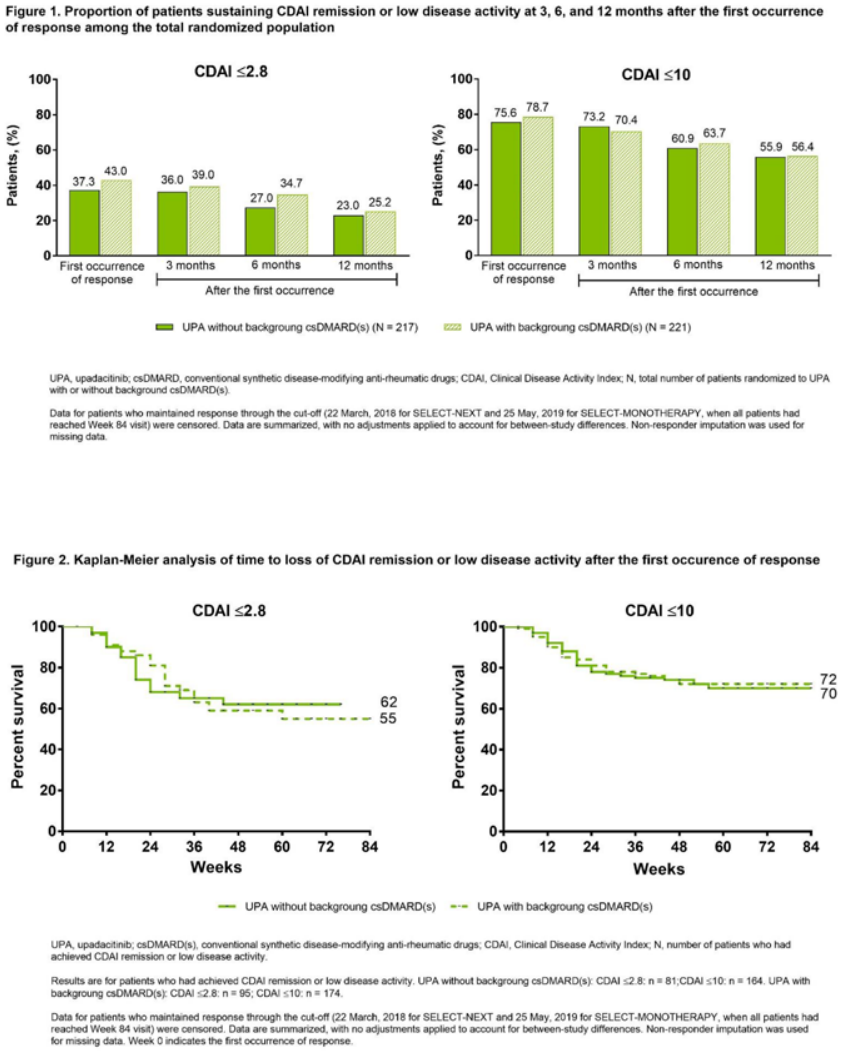

Disclosure of Interests: : Arthur Kavanaugh Grant/research support from: Abbott, Amgen, AstraZeneca, BMS, Celgene Corporation, Centocor-Janssen, Pfizer, Roche, UCB - grant/research support, Maya H Buch Grant/ research support from: Pfizer, Roche, and UCB, Consultant of: Pfizer; AbbVie; Eli Lilly; Gilead Sciences, Inc.; Merck-Serono; Sandoz; and Sanofi, Bernard Combe Grant/research support from: Novartis, Pfizer, Roche-Chugai, Consultant of: AbbVie; Gilead Sciences, Inc.; Janssen; Eli Lilly and Com pany; Pfizer; Roche-Chugai; Sanofi, Speakers bureau: Bristol-Myers Squibb Gilead Sciences, Inc.; Eli Lilly and Company; Merck Sharp \& Dohme; Pfizer Roche-Chugai; UCB, Louis Bessette Grant/research support from: AbbVie, Amgen, Bristol-Myers Squibb, Celgene, Eli Lilly, Janssen, Merck, Novartis, Pfizer, Roche, Sanofi, UCB Pharma, Consultant of: AbbVie, Amgen, Bristol-Myers Squibb, Celgene, Eli Lilly, Janssen, Merck, Novartis, Pfizer, Roche Sanofi, UCB Pharma, Speakers bureau: AbbVie, Amgen, Bristol-Myers Squibb, Celgene, Eli Lilly, Janssen, Merck, Novartis, Pfizer, Sanofi, In-Ho Song Shareholder of: AbbVie Inc., Employee of: AbbVie Inc., Yanna Song Shareholder of: AbbVie Inc., Employee of: AbbVie Inc., Jessica Suboticki Shareholder of: AbbVie Inc., Employee of: AbbVie Inc., Peter Nash Grant/ research support from: AbbVie, Bristol-Myers Squibb, Celgene, Eli Lilly and Company, Gilead, Janssen, MSD, Novartis, Pfizer Inc, Roche, Sanofi UCB, Consultant of: AbbVie, Bristol-Myers Squibb, Celgene, Eli Lilly, Gilead, Janssen, MSD, Novartis, Pfizer Inc, Roche, Sanofi, UCB, Speakers bureau: AbbVie, Bristol-Myers Squibb, Celgene, Eli Lilly, Gilead, Janssen, MSD Novartis, Pfizer Inc, Roche, Sanofi, UCB DOI: 10.1136/annrheumdis-2020-eular.1233

\section{THU0208 AFFECTING COMMON BIOLOGICAL PROCESSES OR DISPARATE?: COMPARISON OF GENE- EXPRESSION MODIFICATION PROFILES AMONG TARGETING IL-6 AND TARGETING SPECIFIC JAK TREATMENTS.}

Y. Koyama ${ }^{1}$, Y. Sato ${ }^{2}$, M. Sakamoto ${ }^{1}$, H. lijima ${ }^{2}$, T. Higuchi ${ }^{1} .{ }^{1}$ Japanese Red Cross Okayama Hospital, Division of Rheumatology, Center for Autoimmune Diseases, Okayama, Japan; ${ }^{2}$ DNA Chip Research Inc, Tokyo, Japan

Background: After accumulation of evidences, it is recognized that inhibition of IL-6 signaling is one of the most established strategies for rheumatoid arthritis (RA) treatment. Tocilizumab (TCZ), an anti-IL6 receptor monoclonal antibody, is the pioneer which blocks IL-6 signaling by preventing IL-6 from binding to both membrane-bound and soluble receptors. Tofacitinib (TOF) inhibits Janus kinase (JAK) 1, JAK3 and, to a lesser extent, JAK2. Recently, Baricitinib (BAR), JAK 1 and JAK2 selective kinase inhibitor, were also approved to treat RA. These JAK inhibitors are known to inhibit cytokine signaling including interleukin (IL)-6. It is very important for clinicians to know whether these treatments affect common biological processes or disparate, because it will provide a rationale for switching each other if one of these treatments resulted in lack of efficacy.

Objectives: To compare the gene-expression modification profiles among TOF BAR and TCZ treatments.

Methods: Total of 38 RA cases were analyzed, including TOF $(n=15$ : $6-20 \mathrm{mg} / \mathrm{d})$, BAR $(\mathrm{n}=10: 2-4 \mathrm{mg} / \mathrm{d})$ and TCZ $(\mathrm{n}=13: 8 \mathrm{mg} / \mathrm{kg} / 4 \mathrm{w}$ or $163 \mathrm{mg} / 2 \mathrm{w})$ treatment groups. Peripheral blood was drawn at just before (pre) and 3 months after (post) these treatments. Total RNAs were then extracted with using PAXgene miRNA kit. After constructing single-stranded, strand-specific libraries, multiplex sequencing was done. After quantifying the expressions of transcripts, differentially expressed genes (DEGs) were selected by paired comparison (post vs. pre), setting thresholds at 2-fold change up/ down and less than $\mathrm{P}=0.05$ in paired T-test. And then, hierarchical clustering analysis and enrichment analysis using gene ontology (GO) terms were performed.

Results: From the comparison of post- vs. pre-treatment of TOF, BAR and TCZ, the 120 (up-regulated $=25 /$ down-regulated $=95), 62($ up $=20 /$ down $=42)$ and 193 (up=54/ down=139) genes were selected as DEGs respectively. It seems to be discrete depending on the treatment, because overlapped genes were only $1.0 \%$ in up-regulated and $5.7 \%$ in down-regulated genes. The hierarchical clustering with expression profiles of these DEGs showed major 4 clusters. $92.3 \%$ of TCZ and $70 \%$ of BAR cases were segregated into $1^{\text {st }}$ and $3^{\text {rd }}$ clusters respectively, while those of TOF cases fell into $2^{\text {nd }}$ and $4^{\text {th }}$ clusters. Disparate GO terms were enriched in each DEGs group. For example genes relevant to viral defense including 'response to type I interferon (IFN)' were suppressed in TOF group. Meanwhile, down regulation of genes involved in phosphorylation process including 'IL-7 signaling' seemed to be significant in BAR group. It is noteworthy that terms related to wound healing such as 'platelet activation' were enriched in the down-regulated genes of TCZ group. 
Conclusion: It is speculated that the downstream biological cascade for TOF, BAR and TCZ treatment might be shared, as IL- 6 signaling is mediated by JAK1/ JAK2/TYK2 activation. However, the influence of these treatments over the transcriptome in the peripheral blood seems to be disparate. Enrichment analysis using GO terms also indicated that different biological processes were involved in the effect of each treatment. Our findings will support a rationale for switching each other if one of these treatments resulted in lack of efficacy. An increased risk of herpes zoster by a treatment with JAK inhibitors has been well recognized. It makes sense because IFN signaling is also mediated by JAK/STAT pathway. On the other hand, we have experienced a case with exacerbation of skin ulcer during TCZ treatment despite the activity of RA was absolutely under control. It is accounted for by the suppression of genes involved in wound healing after TCZ treatment.

Disclosure of Interests: : Yoshinobu Koyama Grant/research support from: Eli-Lilly and Mochida., Speakers bureau: BMS, Ayumi, Chugai, Ono, Mitsubishi Tanabe, Abbvie and Eisai., Yoshiharu Sato: None declared, Moe Sakamoto: None declared, Hiroshi lijima: None declared, Toshie Higuchi: None declared

DOI: 10.1136/annrheumdis-2020-eular.3831

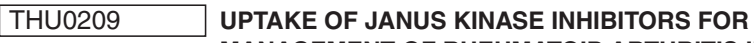 MANAGEMENT OF RHEUMATOID ARTHRITIS IN AUSTRALIA}

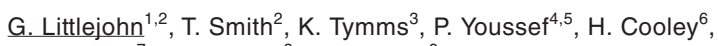
S. Ciciriello ${ }^{7}$, D. Mathers ${ }^{8}, \mathrm{H}$. Griffiths ${ }^{9}$ on behalf of the OPAL Consortium. ${ }^{1}$ Monash Rheumatology, Melbourne, Australia; ${ }^{2}$ OPAL Rheumatology Ltd, Sydney, Australia; ${ }^{3}$ Canberra Rheumatology, Canberra, Australia; ${ }^{4}$ University of Sydney, Sydney, Australia; ${ }^{5}$ Royal Prince Alfred Hospital, Sydney, Australia; ${ }^{6}$ Hobart Private Hospital, Hobart, Australia;

${ }^{7}$ Royal Melbourne Hospital, Melbourne, Australia; ${ }^{8}$ Georgetown Rheumatology, Newcastle, Australia; ${ }^{9}$ Barwon Rheumatology Service, Geelong, Australia

Background: JAK inhibitors (JAKi) are oral tsDMARDs with a different mode of action (MOA) to both oral cs- and parenteral bDMARDs. In Australia the cost of $\mathrm{b} /$ tsDMARDs for treatment of RA is subsidized if the patient has documented high levels of clinical/laboratory disease activity and has not responded to a pre-specified combination of csDMARDs, including MTX. Once eligible for subsidy the clinician can prescribe the b/tsDMARD deemed most clinically appropriate.

Objectives: To determine the patterns of use and reasons for initiation and discontinuation of JAKi in real-world rheumatology practice in Australia.

Methods: Deidentified clinical data were sourced from the OPAL dataset, which is collected in a custom-built electronic medical record at the time of the consultation ${ }^{1}$ by 94 rheumatologists in Australia, representing one third of Australian clinical rheumatologists. Data from patients $>18$ years with a diagnosis of RA who commenced a b/tsDMARD between Jan-2007 and Sept-2019 were included in the analysis. Tableau ${ }^{\circledR}$ was used to display data on medication initiation and cessation dates, and reasons for starting and stopping b/tsDMARDs, which is recorded at the time of the decision.

Results: At Sept 2019, there were 45,317 patients with RA in the data set, with $27 \%$ prescribed b/tsDMARDs. Of patients currently on treatment at Sept 2019 , 53\% were receiving a TNFi and $21 \%$ a JAKi, with the remainder receiving tocilizumab, abatacept or rituximab. Of patients who commenced their current treatment after JAKi's become available in Sept 2015, 46\% were treated with a TNFi, and $32 \%$ were treated with a JAKi. Tofacitinib (TOF) has been the most prescribed b/tsDMARD since Sept 2015 with $22 \%$ of all initiations; however, since baricitinib (BARI) became available in Sept 2018, it has taken over as the preferred JAKi with $24 \%$ of new initiations compared to $14 \%$ for TOF. From Sept 2018-Sept 2019 etanercept and adalimumab were the most commonly prescribed agents in first line, followed by TOF then BARI; however, BARI was the most prescribed agent in lines 2-6+ (figure 1). The main clinician-listed reason for choice of TOF was MOA in $54 \%$, efficacy compared with alternatives in $30 \%$, mode of administration in $7 \%$, efficacy as monotherapy in $7 \%$, and safety in $1 \%$. BARI was chosen for MOA in $35 \%$, efficacy compared with alternatives in $38 \%$, mode of administration in $12 \%$, efficacy as monotherapy in $12 \%$, and safety in $1 \%$. The main reasons for stopping TOF were lack of efficacy (34\%), better alternative (25\%) and adverse reaction (13\%); those for BARI were lack of efficacy (35\%) and adverse reaction (25\%) which is consistent with the rates observed in the first 12-months of clinical experience with TOF, and better alternative $(12 \%)$. Patient non-adherence was listed in $1 \%$ and $2 \%$ of cessations for TOF and BARI, respectively. $45 \%$ of patients discontinuing a JAKi in first line switched to a TNFi in second line, and $40 \%$ switched to another JAKi, citing lack of efficacy, adverse reaction, and better alternative as the reason for switching.

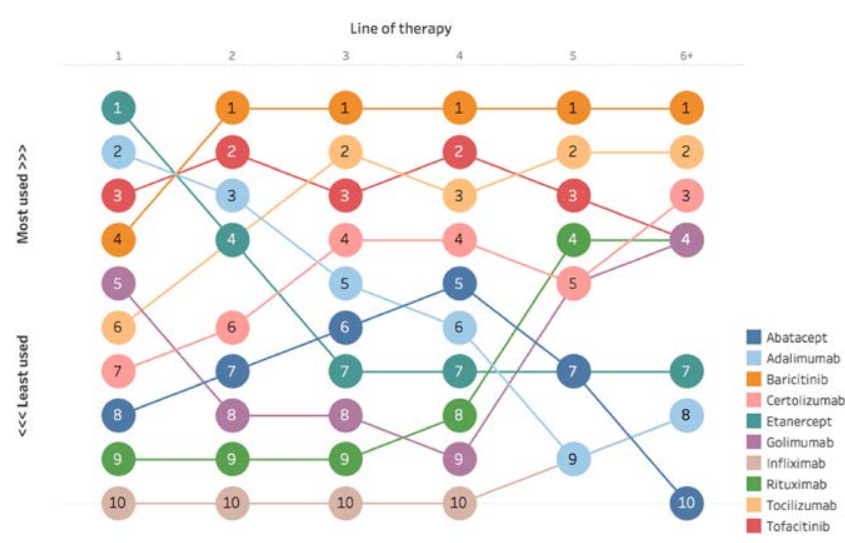

Figure 1. Rank of new initiations by line of therapy (Sept 2018-Sept 2019)

Conclusion: There has been significant and sustained uptake of JAKi for the management of RA in Australia. MOA and perceived efficacy rate much higher than mode of administration for clinicians when selecting a JAKi. Clinical outcomes and persistence following JAKi cycling requires further investigation.

References: Littlejohn GO, Tymms KE, Smith T, Griffiths HT. Using big data from real-world Australian rheumatology encounters to enhance clinical care and research. Clin Exp Rheum Nov 2019

Disclosure of Interests: None declared

DOI: 10.1136/annrheumdis-2020-eular.5055

\begin{tabular}{|l|l}
\hline THU0210 & EARLY DISCONTINUATION OF TOFACITINIB \\
& IN PATIENTS WITH RHEUMATOID ARTHRITIS \\
& CO-TREATED WITH RIFAMPIN FOR LATENT \\
& TUBERCULOSIS: RESULTS FROM THE REAL-WORLD \\
& DATA
\end{tabular}

S. H. Nam ${ }^{1}$, S. J. Choi ${ }^{1}$, J. S. Lee ${ }^{1}$, J. S. Oh ${ }^{2}$, S. Hong ${ }^{1}$, W. J. Seo ${ }^{3}$, C. K. Lee ${ }^{1}$, B. Yoo ${ }^{1}$, Y. G. Kim ${ }^{1} .{ }^{1}$ University of Ulsan College of Medicine, Asan Medical Center, Division of Rheumatology, Department of Internal Medicine, Seoul, Korea, Rep. of (South Korea); ${ }^{2}$ Asan Medical Center, Department of Biomedical Informatics, Seoul, Korea, Rep. of (South Korea); ${ }^{3}$ Veterans Health Service Medical Center, Department of Internal Medicine, Seoul, Korea, Rep. of (South Korea)

Background: Rheumatoid arthritis (RA) patients need to undergo screening and receive treatment for latent tuberculosis infection (LTBI) before starting tofacitinib, which is primarily metabolized by cytochrome P450 (CYP) 3A4. Among chemoprophylactic agents, rifampin is known to be a potent CYP3A4 inducer; therefore, it is expected to decrease the efficacy of tofacitinib. However, tofacitinib and rifampin have been co-administered practically because of the short duration of chemoprophylaxis.

Objectives: The aim of this study was to determine the efficacy of tofacitinib on co-administration with rifampin.

Methods: Biologic-naïve RA patients treated with tofacitinib were selected, and electronic medical reports were reviewed retrospectively. All patients underwent screening for LTBI before starting tofacitinib, and patients with positive results were treated to prevent progression to active tuberculosis. To evaluate the efficacy of tofacitinib with or without rifampin, the discontinuation rates of tofacitinib were examined during the first 6 months. Kaplan-Meier analysis was used to construct cumulative discontinuation curves, and comparisons were performed using the log-rank test.

Results: Among 81 patients who started tofacitinib, 21 (25.9\%) were LTBI-positive and $18(22.2 \%)$ were administered rifampin concomitantly with tofacitinib. The median follow-up time was 6 months in both patients who received rifampin (interquartile range $[\mathrm{IQR}] 2.21,6.00$ ) and those who did not receive rifampin (IQR $5.97,6.00)(p=0.083)$. There were no significant differences between patients who received rifampin and those who did not receive rifampin in all baseline characteristics, except the swollen joint count $(3.00[1.75,5.25]$ vs. $5.00[4.00$, 7.00]; $p=0.025$ ), at the time of starting tofacitinib. In patients who received rifampin at the time of starting tofacitinib, the mean duration of co-administration was $47.00 \pm 23.54$ days (median 56; IQR 28.75, 59.00). During follow-up, 14 of the 81 patients (17.3\%) discontinued tofacitinib. As shown in the Figures 1 and 2 , the discontinuation rate of tofacitinib within the first 6 months was significantly higher among patients who received rifampin for LTBI than among those who did not receive rifampin (lack of efficacy: $24.7 \%$ vs. $5.1 \%, p=0.008$; all causes: $38.9 \%$ vs. $11.2 \%, p=0.002$ ). Seven patients discontinued tofacitinib because of 